\title{
Fault detection and isolation in aircraft gas turbine engines. Part 1: underlying concept
}

S Gupta, A Ray*, S Sarkar, and M Yasar ${ }^{\dagger}$

Department of Mechanical Engineering, The Pennsylvania State University, University Park, Pennsylvania, USA

The manuscript was received on 10 November 2007 and was accepted after revision for publication on 1 February 2008.

DOI: 10.1243/09544100JAERO311

\begin{abstract}
Degradation monitoring is of paramount importance to safety and reliability of aircraft operations and also for timely maintenance of its critical components. This two-part paper formulates and validates a novel methodology of degradation monitoring of aircraft gas turbine engines with emphasis on detection and isolation of incipient faults. In a complex system with multiple interconnected components (e.g. an aircraft engine), fault isolation becomes a crucial task because of possible input-output and feedback interactions among the individual components.

This paper, which is the first of two parts, presents the underlying concepts of fault detection and isolation (FDI) in complex dynamical systems. The FDI algorithms are formulated in the setting of symbolic dynamic filtering (SDF) that has been recently reported in literature. The underlying concept of SDF is built upon the principles of symbolic dynamics, statistical pattern recognition, and information theory. In addition to abrupt large faults, the SDF-based algorithms are capable of detecting slowly evolving anomalies (i.e. deviations from the nominal behaviour) based on analysis of time series data of critical process variables of different engine components. The second part, which is a companion paper, validates the concept, laid out in the first part, on the simulation test bed of a generic two-spool turbofan aircraft engine model for detection and isolation of incipient faults.
\end{abstract}

Keywords: aircraft propulsion, gas turbine engines, fault detection and isolation, statistical pattern recognition

\section{INTRODUCTION}

Performance and reliability of aircraft gas turbine engines gradually deteriorate over the service life due to degradation of the gas path components such as fan, compressor, combustor, and turbines. Common causes of degradation of the gas path components include compressor fouling, increase of the blade-tip clearance in the turbine, labyrinth seal leakage, wear and erosion, and corrosion in the hot sections [1]. These physical faults gradually evolve

\footnotetext{
* Corresponding author: Department of Mechanical Engineering, The Pennsylvania State University, 329 Reber Building, University Park, Pennsylvania 16802, USA. email:axr2@psu.edu

${ }^{\dagger}$ Now at: Techno-Sciences, Inc., Beltsville, Maryland, USA
}

over a prolonged period of operation and lead to degradation of the performance parameters, such as thermodynamic efficiency and flow capacity of individual gas-path components. Performance degradation, in turn, causes changes in the macroscopic observable parameters, such as temperature, pressure, rotational speed, and fuel flow rate. Thus, small faults in the engine system can be detected by measuring the changes in these observable parameters through appropriate usage of signal processing and pattern recognition tools $[2,3]$. Along this line, time series data analysis [4] of gradually evolving changes in pertinent gas path parameters provides one of the most powerful tools for degradation monitoring of engine components.

Current state-of-the-art degradation monitoring of aircraft gas turbine engines provides several fault 
detection and isolation (FDI) tools that vary widely in their complexity and applications and are primarily built upon both model-based and sensor-based analyses. A linear model-based method, called gas path analysis (GPA), was introduced in 1967 by Urban [5] and subsequently different modifications of this method were proposed including usage of Kalman filters, extended Kalman filters, and optimal estimate-based methods [6-9]. To incorporate the non-linearities involved in aircraft engine dynamics, a non-linear model-based method combined with conventional optimization methods was first introduced in early 1990s by Stamatis et al. [10]. However, conventional optimization methods have a possibility of convergence to a local minimum; and this shortcoming was addressed in genetic algorithms, first proposed by Zedda and Singh [11]. Earlier Dietz et al. [12] introduced the concept of neural networks for gas turbine diagnostics, which has been widely used subsequently [13]. Applications of expert systems for gas turbine engine fault detection go back to 1980s and include rule-based fuzzy systems by Fuster et al. [14] and Siu et al. [15].

In a complex system consisting of multiple interconnected components (e.g. an aircraft gas turbine engine), a single fault in one component may produce an anomalous output that might serve as the input excitation to other healthy components, and degrade their performance. Therefore, an anomalous response of an engine component could be due to two possible causes: (a) fault in the component, and/or (b) faulty excitation from some other component. In an extreme adverse scenario, gradual evolution of small anomalies (i.e. deviations from the nominal behaviour) in individual components may lead to cascaded faults because of strong inputoutput and feedback interconnections between the engine components and may eventually cause catastrophic failures and forced shutdown of the entire system.

The above discussion evinces the need for analytical tools, based on time series analysis, to generate sufficiently advanced warnings of emerging faults, which is essential for degradation monitoring and prevention of catastrophic failures in aircraft gas turbine engines. Data-driven tools of statistical pattern recognition has been reported for fault detection and estimation in mechanical systems $[\mathbf{1 6}-\mathbf{1 8}]$, which are potentially capable of degradation monitoring in aircraft gas turbine engines. Recently, anomaly detection algorithms have been constructed in the setting of symbolic dynamic filtering (SDF) $[3,19,20]$ that belongs to the class of data-driven statistical pattern recognition and enables compression of information into pattern vectors of low dimension. The concept of SDF is built upon the principles of multiple disciplines including Statistical Mechanics [21, 22], symbolic dynamics [23], statistical pattern recognition [2], and information theory [24].

SDF includes preprocessing of time series data using the wavelet analysis [25], which is well suited for time-frequency analysis of non-stationary signals and enables noise attenuation and reduction of spurious disturbances from the raw time series data without any significant loss of pertinent information [19]. The wavelet-transformed data is partitioned using the maximum entropy principle [19] to generate the symbol sequences. Subsequently, statistical patterns of evolving anomalies are identified from these symbolic sequences through construction of a (probabilistic) finite-state machine [3] that captures the system behaviour by means of information compression. The state probability vectors, which are derived from the respective state transition probability matrices of the finite state machine under the nominal and anomalous conditions, yield the statistical patterns of the evolving anomalies.

SDF-based pattern recognition algorithms have been experimentally validated for real-time execution in different applications, such as electronic circuits [26] and fatigue damage monitoring in polycrystalline alloys $[\mathbf{2 7}, \mathbf{1 7}, \mathbf{1 8}]$. It has been shown that SDF yields superior performance in terms of early detection of anomalies and robustness to measurement noise by comparison with other existing techniques such as principal component analysis (PCA) and artificial neural networks (ANN) $[\mathbf{2 6}, \mathbf{2 7}]$.

The objective of this two-part paper is to develop and validate a methodology for robust FDI in aircraft gas turbine engines. The FDI algorithms are based on analysis of time series data that can be generated from pertinent sensors and/or analytically derived model variables [28]. This paper, which is the first of two parts, presents the underlying concept of SDF for early detection and isolation of incipient faults in multi-component gas turbine engines via comparison of the current pattern and the nominal pattern. The second part, which is a companion paper, validates the above concept, on the simulation test bed of a generic two-spool turbofan aircraft engine model [29-31] for detection and isolation of incipient faults.

The first part is organized in four sections including the present one. Section 2 provides a brief review of SDF. Section 3 presents the concept of FDI in both single-component and multi-component systems. The first part is summarized in section 4 .

\section{REVIEW OF SDF}

This section presents the underlying concepts and salient features of SDF for anomaly detection in complex dynamical systems. While the details are 
reported in previous publications $[\mathbf{3}, \mathbf{1 9}, \mathbf{2 0}]$ in a scattered fashion, the essential concepts of space partitioning, symbol sequence generation, construction of a finite-state machine from the generated symbol sequence and pattern recognition are consolidated here and succinctly described for self-sufficiency and completeness of the paper.

\subsection{Notion of two time scales for anomaly detection}

Degradation monitoring of complex systems (e.g. an aircraft gas turbine engine) is formulated as a twotime-scale problem. The fast time scale is related to the response time of engine gas path dynamics. Over the span of a given time series data sequence generated on a fast time scale, the dynamic behaviour of the engine system is assumed to remain invariant, i.e. the process is quasi-stationary on the fast time scale. In other words, the variations in the behaviour of engine gas path dynamics are assumed to be negligible over the span of a time series data sequence. The slow time scale is related to the time span over which the critical parameters of the engine operation (e.g. thermodynamic efficiency of the compressor and turbine blade-tip clearance) may change and exhibit non-stationary dynamics. That is, the observable nonstationary behaviour of engine gas path dynamics is associated with anomalies evolving at the slow time scale.

The concept of two time scales is illustrated in Fig. 1. In general, a long time span in the fast time scale is a tiny (i.e. several order of magnitude smaller) interval in the slow time scale. For example, evolution of anomalies in an engine, causing a detectable change in the gas path dynamics, occurs on the slow time scale in the order of hundreds of hours of operation; in contrast, the gas path dynamics remain essentially invariant on the fast time scale in the order of tens of seconds. Nevertheless, the notion of fast and slow time scales is dependent on the specific application, loading conditions, and operating environment. From the perspective of degradation monitoring, time series data sets (e.g. compressor outlet gas temperature and pressure, and electronic signals to the actuators) are collected on the fast time scale at different slow time

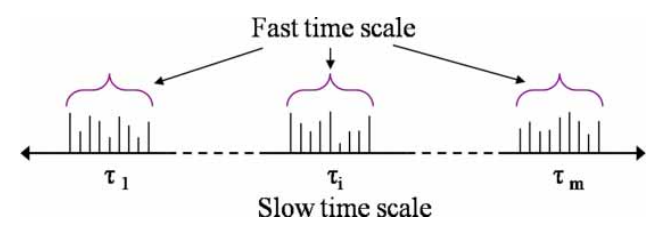

Fig. 1 Pictorial view of the two time scales: (a) slow time scale of anomaly evolution and (b) fast time scale for data acquisition and signal conditioning epochs separated by uniform or non-uniform intervals on the slow time scale.

The following section presents the concept of symbolic dynamics and encoding of time series data in the fast scale.

\subsection{Symbolic dynamics and encoding}

This section briefly describes the concepts of symbolic dynamics for:

(a) encoding nonlinear system dynamics from observed time series data for generation of symbol sequences;

(b) construction of a probabilistic finite state machine (PFSM) from the symbol sequence for generation of pattern vectors as representation of the engine's dynamical characteristics.

The continuously varying finite-dimensional model of a dynamical system, such as an aircraft engine, is usually formulated in the setting of an initial value problem as

$$
\frac{\mathrm{d} \boldsymbol{x}(t)}{\mathrm{d} t}=f\left(\boldsymbol{x}(t), \theta\left(t_{\mathrm{s}}\right)\right) ; \boldsymbol{x}(0)=\boldsymbol{x}_{0}
$$

where $t \in[0, \infty)$ denotes the (fast-scale) time; $\boldsymbol{x} \in \mathbb{R}^{n}$ is the state vector in the phase space; and $\theta \in \mathbb{R}^{\ell}$ is the (possibly anomalous) parameter vector varying in (slow-scale) time $t_{\mathrm{s}}$. The gradual change in the parameter vector $\theta \in \mathbb{R}^{\ell}$ due to possible evolution of anomalies on the slow time scale can alter the system dynamics and hence change the state trajectory.

Let $\Omega \subset \mathbb{R}^{n}$ be a compact (i.e. closed and bounded) region, within which the trajectory of the dynamical system, governed by equation (1), is circumscribed as illustrated in Fig. 2. The region $\Omega$ is partitioned as $\left\{\Phi_{0}, \ldots, \Phi_{|\Sigma|}\right\}$ consisting of $|\Sigma|$ mutually exclusive (i.e. $\Phi_{j} \cap \Phi_{k}=\emptyset \quad \forall j \neq k$ ), and exhaustive (i.e. $\bigcup_{j=0}^{|\Sigma|} \Phi_{j}=\Omega$ ) cells, where $\Sigma$ is the symbol alphabet that

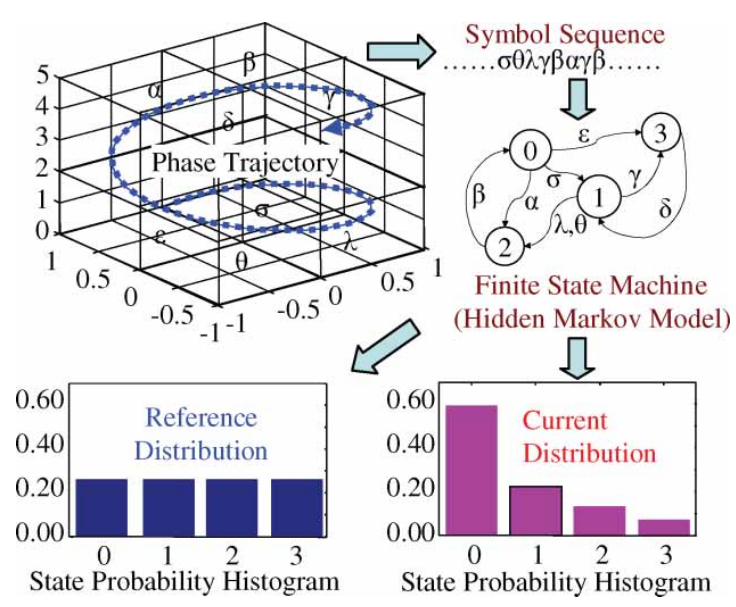

Fig. 2 Concept of symbolic dynamic filtering 
labels the partition cells. A trajectory of the dynamical system is described by the discrete time series data as: $\left\{\mathbf{x}_{0}, \mathbf{x}_{1}, \mathbf{x}_{2}, \ldots\right\}$, where each $\mathbf{x}_{i} \in \Omega$. The trajectory passes through or touches one of the cells of the partition; accordingly the corresponding symbol is assigned to each point $\boldsymbol{x}_{i}$ of the trajectory as defined by the mapping $\mathcal{M}: \Omega \rightarrow \Sigma$. Therefore, a sequence of symbols is generated from the trajectory starting from an initial state $\boldsymbol{x}_{0} \in \Omega$, such that

$$
\boldsymbol{x}_{0} \longrightarrow s_{0} s_{1} s_{2} \ldots s_{j} \ldots
$$

where $s_{k} \triangleq \mathcal{M}\left(\mathbf{x}_{k}\right)$ is the symbol generated at the (fast scale) instant $k$. The symbols $s_{k}, k=0,1, \ldots$ are identified by an index set $\mathcal{I}: \mathbb{Z} \rightarrow\{0,1,2, \ldots|\Sigma|-1\}$, i.e. $\mathcal{I}(k)=i_{k}$ and $s_{k}=\sigma_{i_{k}}$ where $\sigma_{i_{k}} \in \Sigma$. Equivalently, equation (2) is expressed as

$$
\boldsymbol{x}_{0} \longrightarrow \sigma_{i_{0}} \sigma_{i_{1}} \sigma_{i_{2}} \ldots \sigma_{i_{j}} \ldots
$$

The mapping in equations (2) and (3) is called symbolic dynamics as it attributes a legal (i.e. physically admissible) symbol sequence to the system dynamics starting from an initial state. The partition is called a generating partition of the phase space $\Omega$ if every legal (i.e. physically admissible) symbol sequence uniquely determines a specific initial condition $\boldsymbol{x}_{0}$. In other words, every (semi-infinite) symbol sequence uniquely identifies one continuous space orbit [32].

Symbolic dynamics may also be viewed as coarse graining of the phase space, which is subjected to (possible) loss of information resulting from granular imprecision of partitioning boxes. However, the essential robust features (e.g. periodicity and chaotic behaviour of an orbit) are expected to be preserved in the symbol sequences through an appropriate partitioning of the phase space [33].

Figure 2 pictorially elucidates the concepts of partitioning a finite region of the phase space and the mapping from the partitioned space into the symbol alphabet, where the symbols are indicated by Greek letters (e.g. $\alpha, \beta, \gamma, \delta, \ldots$ ). This represents a spatial and temporal discretizations of the system dynamics defined by the trajectories. Figure 2 also shows conversion of the symbol sequence into a finite-state machine and generation of the state probability vectors at the current and the reference conditions. The states of the finite state machine and the histograms in Fig. 2 are indicated by numerics (i.e. $0,1,2$, and 3); the necessary details are provided in section 2.4. Although the theory of phase-space partitioning is well developed for one-dimensional mappings [32], very few results are known for two and higher dimensional systems. Furthermore, the state trajectory of the system variables may be unknown in case of systems for which a model as in equation (1) is not known or is difficult to obtain. As such, as an alternative, the time series data set of selected observable outputs can be used for symbolic dynamic encoding (see following section 2.3 for further details). In general, the time series data can be generated from the available sensors and/or from analytically derived model variables [28].

\subsection{Wavelet space partitioning}

As described earlier, a crucial step in SDF is partitioning of the phase space for symbol sequence generation [34]. Several partitioning techniques have been reported in literature for symbol generation $[4,35]$, primarily based on symbolic false nearest neighbours (SFNN). These techniques rely on partitioning the phase space and may become cumbersome and extremely computation-intensive if the dimension of the phase space is large. Moreover, if the time series data is noise-corrupted, then the symbolic false neighbours would rapidly grow in number and require a large symbol alphabet to capture the pertinent information on the system dynamics. Therefore, symbolic sequences as representations of the system dynamics should be generated by alternative methods because phase-space partitioning might prove to be a difficult task in the case of high dimensions and presence of noise. The wavelet transform [25] largely alleviates these shortcomings and is particularly effective with noisy data from high-dimensional dynamical systems [19]. As such, this paper has used a waveletbased partitioning approach $[\mathbf{3}, \mathbf{1 9}]$ for construction of symbol sequences from time series data.

In wavelet-based partitioning approach, time series data are first converted to wavelet domain, where wavelet coefficients are generated at different time shifts. The choice of the wavelet basis function and wavelet scales depends on the time-frequency characteristics of individual signals. Guidelines for selection of basis functions and scales are reported in literature [19].

The wavelet space is partitioned with alphabet size $|\Sigma|$ into segments of coefficients on the ordinate separated by horizontal lines. The choice of $|\Sigma|$ depends on specific experiments, noise level and also the available computation power. A large alphabet may be noisesensitive while a small alphabet could miss the details of signal dynamics [19]. The partitioning is done such that the regions with more information are partitioned finer and those with sparse information are partitioned coarser. This is achieved by maximizing the Shannon entropy [24], which is defined as

$$
S=-\sum_{i=0}^{|\Sigma|-1} p_{i} \log \left(p_{i}\right)
$$

where $p_{i}$ is the probability of a data point to be in the $i$ th partition segment. Uniform probability 


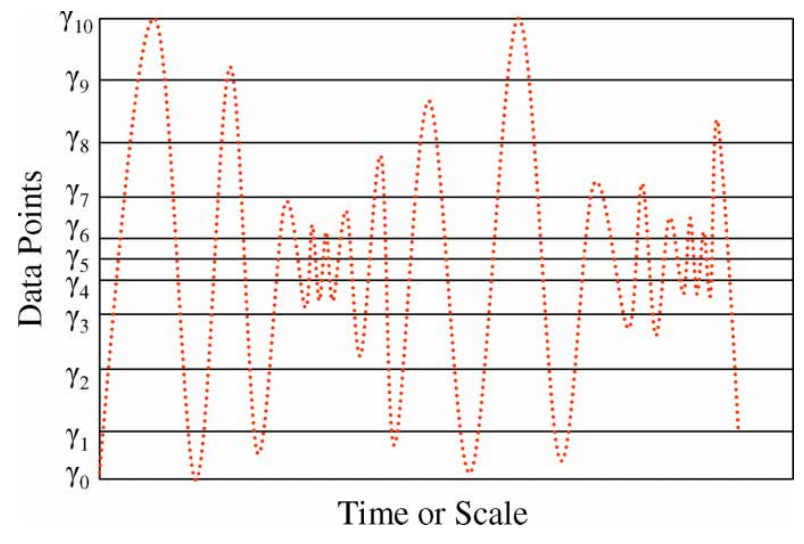

Fig.3 An example of maximum entropy partitioning

distribution, i.e. $p_{i}=1 /|\Sigma|$ for $i=1,2, \ldots,|\Sigma|$, is a consequence of maximum entropy partitioning [19]. In the illustrative example of Fig. 3 , the partitioning contains 10 cells (i.e. line intervals in this case), where the size of the cells is smaller for regions with higher density of data points to ensure an unbiased partition such that each cell is allocated equal number of visits at the nominal condition.

Each partition segment is labelled by a symbol from the alphabet $\Sigma$ and accordingly the symbol sequence is generated from the wavelet coefficients. The structure of the partition is fixed at the nominal condition, which serves as the reference frame for symbol sequence generation from time series data at anomalous condition(s).

\subsection{PFSM and pattern recognition}

Once the symbol sequence is obtained, the next step is the construction of a PFSM and calculation of the respective state probability vector as depicted in the lower part of Fig. 2 by the histograms. The partitioning (see Fig. 3 ) is performed at the slow time epoch $t_{0}$, i.e. the nominal condition that is chosen to be the healthy state having no anomalies.

A PFSM is then constructed at the nominal condition, where the states of the machine are defined corresponding to a given alphabet set $\Sigma$ and window length $D$. The alphabet size $|\Sigma|$ is the total number of partition segments while the window length $D$ is the length of consecutive symbol words [3], which are chosen as all possible words of length $D$ from the symbol sequence. Each state belongs to an equivalence class of symbol words of length $D$, which is characterized by a word of length $D$ at the leading edge. Therefore, the number $n$ of such equivalence classes (i.e. states) is less than or equal to the total permutations of the alphabet symbols within words of length $D$. That is, $n \leqslant|\Sigma|^{D}$; some of the states may be forbidden, i.e. these states have zero probability of occurrence. For example, if $\Sigma=\{\alpha, \beta\}$, i.e. $|\Sigma|=2$ and if $D=2$, then

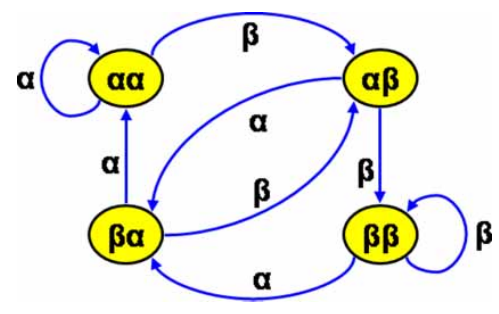

Fig. 4 Example of finite state machine with $D=2$ and $\Sigma=\{\alpha, \beta\}$

the number of states is $n \leqslant|\Sigma|^{D}=4$; and the possible states are words of length $D=2$, i.e. $\alpha \alpha, \alpha \beta, \beta \alpha$, and $\beta \beta$, as shown in Fig. 4 .

The choice of $|\Sigma|$ and $D$ depends on specific applications and the noise level in the time series data as well as on the available computation power and memory availability. As stated earlier, a large alphabet may be noise-sensitive and a small alphabet could miss the details of signal dynamics. Similarly, while a larger value of $D$ is more sensitive to signal distortion, it would create a much larger number of states requiring more computation power and increased length of the data sets. In the second part [36] of this two-part paper, the analysis of time series data sets is done using the window length equal to $D=1$; consequently, the set of states $Q$ is equivalent to the symbol alphabet $\Sigma$. With the selection of the parameters $D=1$ and $|\Sigma|=8$, the PFSM has $n=8$ states. With this choice of parameters, the SDF algorithm is shown to be capable of early detection of anomalies and incipient faults in the gas turbine engine simulation [36]. However, other applications such as two-dimensional image processing, may require larger values of the parameter $D$ and hence possibly larger number of states in the PFSM.

Using the symbol sequence generated from the time series data, the state machine is constructed on the principle of sliding block codes [23]. The window of length $D$ on a symbol sequence is shifted to the right by one symbol, such that it retains the most recent $(D-1)$ symbols of the previous state and appends it with the new symbol at the extreme right. The symbolic permutation in the current window gives rise to a new state. The PFSM constructed in this fashion is called the $D$-Markov machine [3], because of its Markov properties.

\section{Definition 1}

A symbolic stationary process is called $D$-Markov if the probability of the next symbol depends only on the previous $D$ symbols, i.e. $P\left(s_{j} \mid s_{j-1} \ldots s_{j-D} s_{j-D-1} \ldots\right)=$ $P\left(s_{j} \mid s_{j-1} \ldots s_{j-D}\right)$.

The finite state machine constructed above has $D$-Markov properties because the probability of 
occurrence of symbol $\sigma \in \Sigma$ on a particular state depends only on the configuration of that state, i.e. the previous $D$ symbols. The states of the machine are marked with the corresponding symbolic word permutation and the edges joining the states indicate the occurrence of a symbol $\sigma$. The occurrence of a symbol at a state may keep the machine in the same state or move it to a new state.

\section{Definition 2}

Let $\Xi$ be the set of all states of the finite state machine. Then, the probability of occurrence of symbols that cause a transition from state $\xi_{j}$ to state $\xi_{k}$ under the mapping $\delta: \Xi \times \Sigma \rightarrow \Xi$ is defined as

$$
\pi_{j k}=P\left(\sigma \in \Sigma \mid \delta\left(\xi_{j}, \sigma\right) \rightarrow \xi_{k}\right) ; \quad \sum_{k} \pi_{j k}=1
$$

Thus, for a $D$-Markov machine, the irreducible stochastic matrix $\Pi \equiv\left[\pi_{i j}\right]$ describes all transition probabilities between states such that it has at most $|\Sigma|^{D+1}$ nonzero entries. The definition above is equivalent to an alternative representation such that

$$
\pi_{j k} \equiv P\left(\xi_{k} \mid \xi_{j}\right)=\frac{P\left(\xi_{j}, \xi_{k}\right)}{P\left(\xi_{j}\right)}=\frac{P\left(\sigma_{i_{0}} \cdots \sigma_{i_{D-1}} \sigma_{i_{D}}\right)}{P\left(\sigma_{i_{0}} \cdots \sigma_{i_{D-1}}\right)}
$$

where the corresponding states are denoted by $\xi_{j} \equiv$ $\sigma_{i_{0}} \cdots \sigma_{i_{D-1}}$ and $\xi_{k} \equiv \sigma_{i_{1}} \cdots \sigma_{i_{D}}$. This phenomenon is a consequence of the PFSM construction based on the principle of sliding block codes described above, where the occurrence of a new symbol causes a transition to another state or possibly the same state.

For computation of the state transition probabilities from a given symbol sequence at a particular slow time epoch, a window of length $D$ is moved by counting occurrences of symbol blocks $\sigma_{i_{0}} \cdots \sigma_{i_{D-1}} \sigma_{i_{D}}$ and $\sigma_{i_{0}} \cdots \sigma_{i_{D-1}}$, which are respectively denoted by $N\left(\sigma_{i_{0}} \cdots \sigma_{i_{D-1}} \sigma_{i_{D}}\right)$ and $N\left(\sigma_{i_{0}} \cdots \sigma_{i_{D-1}}\right)$. Note that if $N\left(\sigma_{i_{0}} \cdots \sigma_{i_{D-1}}\right)=0$, then the state $\sigma_{i_{0}} \cdots \sigma_{i_{D-1}} \in \Xi$ has zero probability of occurrence. For $N\left(\sigma_{i_{0}} \cdots \sigma_{i_{D-1}}\right) \neq 0$, the estimates of the transitions probabilities are then obtained by these frequency counts as follows

$$
\pi_{j k} \approx \frac{N\left(\sigma_{i_{0}} \cdots \sigma_{i_{D-1}} \sigma_{i_{D}}\right)}{N\left(\sigma_{i_{0}} \cdots \sigma_{i_{D-1}}\right)}
$$

where the criterion for convergence of the estimated $\pi_{j k}$, is given in the following section 2.5 as a stopping rule for frequency counting.

The symbol sequence generated from the time series data at the reference condition, set as a benchmark, is used to compute the state transition matrix $\Pi$ using equation (7). The left eigenvector $\boldsymbol{q}$ corresponding to the unique unit eigenvalue of the irreducible stochastic matrix $\Pi$ is the probability vector whose elements are the stationary probabilities of the states belonging to $\Xi[3]$. Similarly, the state probability vector $\boldsymbol{p}$ is obtained from time series data at a (possibly) faulty condition. The partitioning of time series data and the state machine structure should be the same in both cases but the respective state transition matrices could be different. The probability vectors $\boldsymbol{p}$ and $\boldsymbol{q}$ are estimates of the respective true probability vectors and are treated as statistical patterns. The terms probability vector and pattern vector are used interchangeably in the sequel.

Pattern changes may take place in dynamical systems due to accumulation of faults and progression of anomalies. The pattern changes are quantified as deviations from the reference pattern (i.e. the probability distribution at the reference condition). The resulting anomalies (i.e. deviations of the evolving patterns from the reference pattern) are characterized by a scalar-valued function, called anomaly measure $\mu$. The anomaly measures are obtained as

$$
\mu \equiv d(\boldsymbol{p}, \boldsymbol{q})
$$

where the $d(\bullet, \bullet)$ is an appropriately defined distance function.

\subsection{Stopping rule for symbol sequence generation}

This section presents a stopping rule that is necessary to find a lower bound on the length of symbol sequence required for parameter identification of the stochastic matrix $\Pi$. The stopping rule $[\mathbf{2 0}, \mathbf{3 7}, \mathbf{3 8}]$ is based on the properties of irreducible stochastic matrices [39]. The state transition matrix, constructed at the $r$ th iteration (i.e. from a symbol sequence of length $\mathrm{r}$ ), is denoted as $\Pi(r)$ that is an $n \times n$ irreducible stochastic matrix under stationary conditions. Similarly, the state probability vector $\boldsymbol{p}(r) \equiv$ $\left[p_{1}(r) p_{2}(r) \cdots p_{n}(r)\right]$ is obtained as

$$
p_{i}(r)=\frac{r_{i}}{\sum_{j=1}^{n} r_{j}}
$$

where $r_{i}$ is the number of symbols in the $i$ th state such that $\sum_{j=1}^{n} r_{i=r}$. The stopping rule makes use of the Perron-Frobenius Theorem [39] to establish a relation between the vector $\boldsymbol{p}(r)$ and the matrix $\Pi(r)$. Since the matrix $\Pi(r)$ is stochastic and irreducible, there exists a unique eigenvalue $\lambda=1$ and the corresponding left eigenvector $\boldsymbol{p}(r)$ (normalized to unity in the sense of absolute sum). The left eigenvector $\boldsymbol{p}(r)$ represents the state probability vector, provided that the matrix parameters have converged after a sufficiently large number of iterations. That is, under the hypothetical arbitrarily long sequences, the following condition is 
assumed to hold

$$
\boldsymbol{p}(r+1)=\boldsymbol{p}(r) \Pi(r) \Rightarrow \boldsymbol{p}(r)=\boldsymbol{p}(r) \Pi(r) \text { as } r \rightarrow \infty
$$

Following equation (9), the absolute error between successive iterations is obtained such that

$$
\|(\boldsymbol{p}(r)-\boldsymbol{p}(r+1))\|_{\infty}=\|\boldsymbol{p}(r)(\mathbf{I}-\Pi(r))\|_{\infty} \leqslant \frac{1}{r}
$$

where $\|\bullet\|_{\infty}$ is the maximum norm of the finitedimensional vector $\bullet$.

To calculate the stopping point $r_{\text {stop }}$, a tolerance of $\eta$, $(0<\eta \ll 1)$ is specified for the relative error such that:

$$
\frac{\|(\boldsymbol{p}(r)-\boldsymbol{p}(r+1))\|_{\infty}}{\|(\boldsymbol{p}(r))\|_{\infty}} \leqslant \eta \quad \forall r \geqslant r_{\text {stop }}
$$

The objective is to obtain the least conservative estimate for $r_{\text {stop }}$ such that the dominant elements of the probability vector have smaller relative errors than the remaining elements. Since the minimum possible value of $\|(\boldsymbol{p}(r))\|_{\infty}$ for all $r$ is $1 / n$, where $n$ is the dimension of $\boldsymbol{p}(r)$, the least of most conservative values of the stopping point is obtained from equations. (11) and (12) as

$$
r_{\text {stop }} \equiv \operatorname{int}\left(\frac{n}{\eta}\right)
$$

where int $(\bullet)$ is the integer part of the real number $\bullet$.

\subsection{Summary of SDF-based pattern recognition}

The SDF method of statistical pattern recognition for anomaly detection is summarized below.

1. Acquisition of time series data from appropriate sensor(s) and/or analytically derived model variables at a reference condition, when the system is assumed to be in the healthy state (i.e. zero anomaly measure).

2. Generation of the wavelet transform coefficients, obtained with an appropriate choice of the wavelet basis and scale [19].

3. Maximum entropy partitioning in the wavelet domain at the nominal condition (see section 2.3) and generation of the corresponding symbol sequence.

4. Construction of the $D$-Markov machine and computation of the state probability vector $\boldsymbol{q}$ at the reference condition.
5. Generation of a time series data sequence at another (possibly) faulty condition and conversion to the wavelet domain to generate the respective symbolic sequence based on the partitioning constructed at the reference condition.

6. Computation of the corresponding state probability vector $\boldsymbol{p}$ using the finite state machine constructed at the reference condition.

7. Computation of scalar anomaly measure $\mu$ (see equation (8)).

Capability of SDF has been demonstrated for anomaly detection at early stages of gradually evolving faults by real-time experimental validation. Application examples include active electronic circuits [26] and fatigue damage monitoring in polycrystalline alloys $[\mathbf{1 7}, \mathbf{1 8}, \mathbf{2 7}]$. It has been shown that SDF yields superior performance in terms of early detection of anomalies and robustness to measurement noise by comparison with other existing techniques such as PCA and ANN [26, 27]. In this regard, major advantages of SDF for small anomaly detection are listed below:

(a) robustness to measurement noise and spurious signals [19];

(b) adaptability to low-resolution sensing due to the coarse graining in space partitions [3];

(c) capability for early detection of anomalies because of sensitivity to signal distortion [27];

(d) real-time execution on commercially available inexpensive platforms $[\mathbf{2 6}, \mathbf{2 7}]$.

\section{FAULT DETECTION AND ISOLATION (FDI)}

This section presents the details of SDF-based FDI for degradation monitoring in multiple-component complex systems with various input-output and feedback interconnections. The aircraft gas turbine engine is one such system, as defined above, that is composed of different interconnected components (e.g. fan, compressor, combustor, high-pressure and low-pressure turbines, nozzle, and afterburner) and the engine control system. The interconnections among different components of an engine system include many thermodynamic, mechanical and electronic links that are arranged in the form of a complex input-output and feedback structure [36]. As described earlier, a single fault in one component may produce an anomalous output that might serve as the input excitation to some other healthy component(s), leading to possible loss of performance and other detrimental effects. For example, a healthy component (e.g. high pressure turbine) may function poorly and yield anomalous outputs if it receives incorrect inputs from its predecessor (e.g. the combustor). On the other hand, the combustor might be receiving faulty inputs from the 
compressor and consequently the other components of the engine system may also malfunction. Similarly, electronic hardware and software faults in the control and instrumentation system could lead to engine malfunction. In essence, an anomalous response of an engine component could be due to one or both of the following causes:

(a) faulty component;

(b) anomalous excitation(s) from some other component(s).

Therefore, in an extreme adverse scenario, gradual evolution of faults in individual components may lead to cascaded failure(s) because of strong interconnections between the engine components, which could eventually cause forced shutdown of the entire system.

In addition to major component faults that may lead to catastrophic failures in aircraft engines, a synergistic combination of a number of small faults may become highly detrimental due to amplification of anomalous responses and because of strong interconnections among the critical components of the engine. From the perspective of degradation monitoring of the engine system, it is important to analyse the local behaviour of its components with the capability of detection and isolation of incipient faults. Accordingly, degradation monitoring of all pertinent components must be conducted simultaneously to arrive at meaningful conclusions on the health status of the engine system.

The procedure for FDI in aircraft gas turbine engines includes integrated dynamic process models of engine components [30] and is constructed in the setting of SDF for pattern recognition and anomaly detection (see section 2). The engine component models are augmented with SDF-based FDI algorithms for real-time execution on inexpensive and commercially available computational platforms. Time series data, generated from available sensors and/or analytically derived model variables for each component, are fed to the FDI algorithms for extraction of the relevant information on the nature and location of faults. Details of implementation on an engine simulation test bed are reported in the second part [36] of this paper.

Detection and isolation of both abrupt and gradually evolving faults are crucial for safe and reliable operation of complex systems with multiple interconnected components. Fault detection algorithms involve identification of off-nominal behavioural patterns that are different from the nominal pattern (i.e. the pattern under healthy conditions). Furthermore, it is necessary to reduce the probability of false alarms due to noise and disturbances as well as possible operational errors. Fault detection normally requires analysis of the data from available sensors and/or analytically derived model variables of the system, which are sensitive to small changes. Fault detection algorithms include signal processing tools for noise reduction and improved performance, and are also supported by the information generated from regular inspection and maintenance scheduling.

Fault isolation is the next critical issue in multicomponent complex systems, which involves identification of the malfunctioning component(s). It is essential to identify the sources of the faults by detecting and isolating the genuinely malfunctioning components. This requires analysis of time series data of local sensors and/or analytically derived model variables of individual components.

The FDI algorithms are constructed in the setting of SDF as presented in Figs 5 and 6. Time series data from the relevant outputs of a component are analysed using the SDF-based algorithms for generation of pattern vectors as explained in section 2 . For each component, a nominal model is constructed such that the model outputs $z$ are statistically similar to the healthy component's actual outputs $y$ under identical input excitation $\boldsymbol{u}$ as illustrated in Fig. 5. Typically, the outputs $y$ are obtained as either sensor data or, if such sensors are unavailable, analytically derived information as functions of other available sensor data. Similarly, the input excitation $\boldsymbol{u}$ consists of sensor data and/or analytically derived information and, in addition, command inputs to the component's actuators,

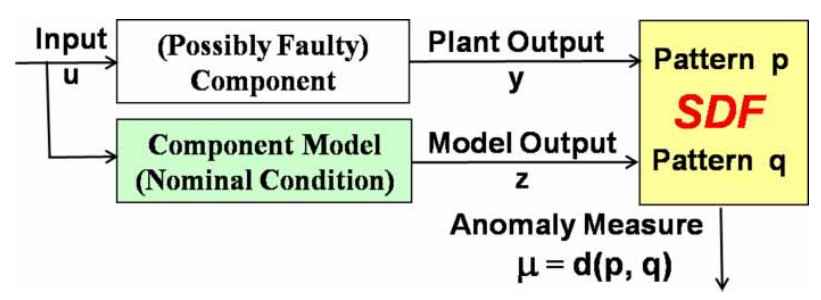

Fig. 5 Fault detection and isolation by comparison with the reference pattern at the nominal condition

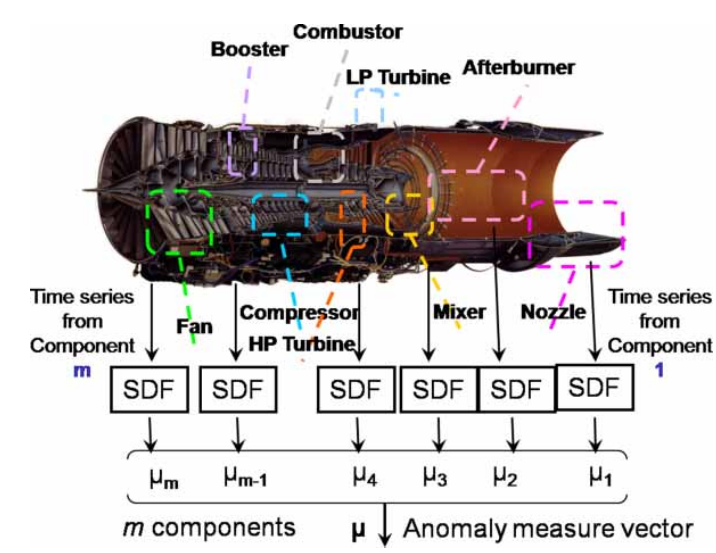

Fig. 6 Fault monitoring in an aircraft gas turbine engine 
if any, which are available in real time as electronic signals from the control computer. For example, if the high-pressure compressor is chosen as a component, its outputs may include the outlet air temperature and air pressure; and the inputs would consist of inlet air temperature, air pressure, and shaft speed of the high-pressure turbine together with the command input to the guide vane actuator. Nevertheless, the choice of components and their input and output is often problem-specific and depends on the user's judgement to some extent.

\section{Remark 1}

Aircraft gas turbine engines are usually provided with interconnected feedback control to maintain the specified performance $[\mathbf{2 9}, \mathbf{3 0}]$ because, in the absence of such control actions, the output variables of a component would deviate from those at the desired operating condition as a result of internal faults or exogenous disturbances. To compensate for these events, the feedback controller modifies the actuator inputs to maintain the specified outputs of the faulty component, if possible, close to the those of the nominal model. Therefore, input excitation to the nominal model should include input command signals of the actuators, if any, which are manipulated by the controller. If the actuator command inputs are modified to compensate for faults and disturbances, then the output of nominal model would deviate from the component output and the resulting anomaly measure would lead to FDI. This issue is discussed in more details in the second part [36] of this paper.

Some of the engine variables, which are not measurable through direct deployment of sensors, may contain sufficient information for FDI. Often these variables can be analytically derived from other available sensor data via physics-based or empirical models [28]. For example, temperature and pressure sensors may not be commercially available for placement at the high pressure turbine inlet although sensors are available for measurement of pressure and temperature at the combustor inlet (i.e. high-pressure compressor exit). For the purpose of FDI, it is possible to obtain an estimate of enthalpy at the high-pressure turbine inlet from the information on other analytically derived and sensor variables. A summary of model equations for the major components of a gas turbine engine is provided in Appendix of the second part of this paper [36].

Let $C=\left\{C_{i}: 1 \leqslant i \leqslant m\right\}$ be the set of all critical components in a multi-component system. Let the input excitation to the $i$ th component $C_{i}$ be denoted by a vector $\boldsymbol{u}_{i}$ and let the corresponding actual output response and the nominal model output response of $C_{i}$ be given by vectors $\boldsymbol{y}_{i}$ and $\boldsymbol{z}_{i}$, respectively. For the component $C_{i}$ at each slow time epoch $t_{k}$, the pattern vectors $\boldsymbol{p}_{i}^{k}$ and $\boldsymbol{q}_{i}^{k}$ are generated from the respective time series data sets $\left\{y_{i}^{k}\right\}$ and $\left\{z_{i}^{k}\right\}$. As seen in Fig. 5, the pattern vector $\boldsymbol{p}_{i}^{k}$ characterizes the health status of $C_{i}$ with reference to the pattern vector $\boldsymbol{q}_{i}^{k}$ that represents the nominal healthy status of $C_{i}$ under the input excitation $\boldsymbol{u}_{i}^{k}$. Following equation (8), the anomaly measure for $C_{i}$ at a time epoch $t_{k}$ is obtained as

$$
\mu_{i}^{k} \equiv d\left(\boldsymbol{p}_{i}^{k}, \mathbf{q}_{i}^{k}\right)
$$

where $d(\bullet, \bullet)$ is an appropriately chosen distance function (Euclidean norm in this case [3]).

The anomaly measure $\mu_{i}^{k}$ would detect a fault if the component $C_{i}$ genuinely malfunctions relative to the nominal condition; otherwise, even if the input to the component $C_{i}$ is anomalous due to a fault in some other component, $\mu_{i}^{k}$ should be insignificantly small provided that the nominal model is a good (statistical) representation of the component $C_{i}$. In this context, the following issues are noteworthy.

1. Point-by-point comparison of data points in time series sets $\boldsymbol{y}_{i}^{k}$ and $\boldsymbol{z}_{i}^{k}$ is not physically meaningful because of the possible phase differences and noise in the signals, thereby necessitating the use of statistical comparison of pattern vectors.

2. Time series data $\boldsymbol{z}_{i}^{k}$ generated from the nominal model of $C_{i}$, could be different at different time epochs because the input excitation $\boldsymbol{u}_{i}^{k}$ to the $C_{i}$ may have different profiles because of occurrence of a fault in some other component.

3. Since the input excitation to both the nominal model and the actual component is identical, an observation of detectable anomaly measure $\mu_{i}^{k}$ for a component $C_{i}$ at time epoch $t_{k}$ (see Fig. 5) would indicate a fault in that component. This procedure facilitates both fault detection and isolation in each component of the system.

Having computed the anomaly measure of individual components, a global picture of the system health could be obtained from the ensemble of generated information. As an illustration, the engine system in Fig. 6 is divided into several components such that each component $C_{i}$ yields the anomaly measure $\mu_{i}^{k}$ at (slow) time epochs $t_{k}$. For $m$ identified components in the engine system, the ensemble of information generated from SDF of individual components is expressed as an $m$-dimensional anomaly measure vector $\boldsymbol{\mu}^{k}=\left[\begin{array}{llll}\mu_{1}^{k} & \mu_{2}^{k} & \cdots & \mu_{m}^{k}\end{array}\right]^{\mathrm{T}}$. The evolution of anomaly measure vector $\mu^{k}$ at the slow time scale determines the behavioural changes occurring in the individual components and their effects on the health of the engine system. With an appropriate decision algorithm (that is problem-specific and depends on the user's needs), this ensemble of information would be capable of isolating both single-component 
and multiple-component faults; results of case studies are presented in the second part [36] of this paper.

Once a component is detected and isolated as faulty, the next task is to identify the range of the fault parameters for prognosis of potential failures as an extension of the work reported in this paper. From this perspective, the task of degradation monitoring is posed as the following two inter-related problems.

1. The forward (or analysis) problem generates behavioural patterns of system evolution due to progression of faults through analysis of the observed time series data at different slow time epochs. Based on this information, the objective here is online identification of the changes in patterns of the engine dynamics due to evolving faults.

2. The inverse (or synthesis) problem identifies the range of component fault parameters to assess the system health based on online observations. The objective here is to infer the anomalies and to estimate the range of the fault parameters on-line from time series data during the engine operation or based on simulation runs in the engine test bed [36] as time evolves. Generation of the anomaly measure information in the forward problem is a predecessor to the inverse problem $[\mathbf{1 8}, 40]$.

The above concept of identifying the fault parameter range has been experimentally validated for fatigue life estimation in polycrystalline alloys $[\mathbf{1 8}]$ and faulty parameter estimation in active electronic circuits [40]. The work is in progress for identification of engine fault parameters and the results are expected to be reported in future publications.

\section{SUMMARY}

This paper, which is the first of two parts, presents the underlying concepts of FDI in complex systems, specifically for aircraft gas turbine engines, that consist of multiple interconnected components. The FDI methodology has been validated on the simulation test bed of a generic two-spool turbofan engine, as reported in the second part [36] of this paper.

The FDI algorithms are formulated in the setting of SDF that has been recently reported in literature $[\mathbf{3}, \mathbf{1 9}]$ and experimentally validated for various applications [26, 27]. The salient features of the proposed FDI methodology are summarized below.

1. Usage of SDF for computation of pattern vectors from the output time series data sets of individual components and their respective nominal models.

2. Comparison of the pattern vector of a component with that of its respective nominal model to generate the anomaly measure which is an indicative of evolving fault(s) in that specific component.
3. Decisions on detection and isolation of evolving fault(s) in individual components.

4. Identification of the fault range in the isolated components via inverse mapping of the respective anomaly measure $[\mathbf{1 8}, \mathbf{4 0}]$.

\section{ACKNOWLEDGEMENTS}

This work has been supported in part by NASA under Cooperative Agreement no. NNX07AK49A and by the US Army Research Laboratory and the US Army Research Office under grant no. W911NF-07-1-0376.

\section{REFERENCES}

1 Razak, A. M. Y. and Carlyle, J. S. An advanced model based health monitoring system to reduce gas turbine ownership cost. ASME TURBO EXPO 2000, Munich, Germany, 2000, paper no. ASME-2000-GT-627.

2 Duda, R. O., Hart, P. E., and Stork, D. G. Pattern classification, 2001 (John Wiley, New York, USA).

3 Ray, A. Symbolic dynamic analysis of complex systems for anomaly detection. Signal Process., 2004, 84(7), 11151130.

4 Kantz, H. and Schreiber, T. Nonlinear time series analysis, 2nd edition, 2004 (Cambridge University Press, Cambridge, UK).

5 Urban, L. A. Gas turbine engine parameter interrelationships, 1967 (HSD UTC, Windsor Locks, CT, USA).

6 Volponi, A. J., DePold, H., Ganguli, R., and Chen, D. The use of Kalman filter and neural network methodologies in gas turbine performance diagnostics: a comparative study. ASME TURBO EXPO 2000, Munich, Germany, 2000, paper no. ASME-2000-GT-547.

7 Kobayashi, T. and Simon, D. L. Application of a bank of Kalman filters for aircraft engine fault diagnostics. TM-2003-212526.10, NASA Glenn Research Center, Cleveland, OH, USA, 2003.

8 Yedavalli, R. K., Shankar, P., Siddiqi, M., and Behbahani, A. Modeling, diagnostics and prognostics of a two-spool turbofan engine. In Proceedings of the 41st AIAA/ASME/SAE/ASEE Joint Propulsion Conference and Exhibit, Tucson, Arizona, 10-13 July 2005, AIAA 2005-4344.

9 Rausch, R., Viassolo, D. E., Kumar, A., Goebel, K., Eklund, N., Brunell, B., and Bonanni, P. Towards inflight detection and accommodation of faults in aircraft engines. In Proceedings of the AIAA 1st Intelligent Systems Technical Conference, Chicago, Illinois, 2004.

10 Stamatis, A., Mathioudakis, K., Smith, M., and Papailiou, K. Gas turbine component fault identification by means of adaptive performance modeling. In 35th International Gas Turbine and Aeroengine Congress and Exposition, Brussels, Belgium, 1990, ASME paper 90-GT-376.

11 Zedda, M. and Singh, R. Gas turbine engine and sensor fault diagnosis using optimization techniques. In Proceedings of the AIAA/ASME/SAE/ASEE Joint Propulsion 
Conference and Exhibit, Los Angeles, California, 1999, AIAA 99-2530, pp. 1-11.

12 Dietz, W. E., Kiech, E. L., and Ali, M. Jet and rocket engine fault diagnosis in real time. J. Neural Netw. Comput., 1989, 1, 5-18.

13 Moller, J. C., Litt, J. S., and Guo, T. H. Neural networkbased sensor validation for turboshaft engines. In the 34th Joint Propulsion Conference cosponsored by AIAA, ASME, SAE, and ASEE, Cleveland, OH, USA, 1998, paper no. AIAA-98-3547.

14 Fuster, P., Ligeza, A., and Martin, A. J. Abductive diagnostic procedure based on an AND/OR/NOT graph for expected behavior: application to a gas turbine. In the 10th International Congress and Exhibition on Condition Monitoring and Diagnostic Engineering management (COMADEM 97), Espoo, Finland, 1997, pp. 511-520.

15 Siu, C., Shen, Q., and Milne, R. TMDOCTOR: a fuzzy rule and case-based expert system for turbomachinery diagnosis. In Proceedings of the 3rd IFAC Symposium on Fault Detection, Supervision and Safety for Technical Processes, Kingston upon Hull, UK, vol. 2, 1997.

16 Grondel, S., Delebarre, C., Assaad, J., Dupuis, J. P., and Reithler, L. Fatigue crack monitoring of riveted aluminium strap joints by Lamb wave analysis and acoustic emission measurement techniques. NDT\&E Int., 2006, 35, 339-351.

17 Gupta, S., Ray, A., and Keller, E. Fatigue damage monitoring by ultrasonic measurements: a symbolic dynamics approach. Int. J. Fatigue, 2007, 29(6), 1100-1114.

18 Gupta, S. and Ray, A. Real-time fatigue life estimation in mechanical systems. Meas. Sci. Technol., 2007, 18(7), 1947-1957.

19 Rajagopalan, V. and Ray, A. Symbolic time series analysis via wavelet-based partitioning. Signal Process., 2006, 86(11), 3309-3320.

20 Gupta, S. and Ray, A. Symbolic dynamic filtering for datadriven pattern recognition. In Pattern recognition: theory and application (Ed. E. A. Zoeller), 2007, ch. 2 (Nova, Science Publisher, Hauppage, New York).

21 Pathria, R. K. Statistical mechanics, 2nd edition, 1996 (Butterworth Heinemann, Oxford, UK).

22 Gupta, S. and Ray, A. Pattern identification using lattice spin systems: a thermodynamic formalism. Appl. Phys. Lett., 2007, 91 (19), 194105.

23 Lind, D. and Marcus, M. An introduction to symbolic dynamics and coding, 1995 (Cambridge University Press, Cambridge, UK).

24 Cover, T. M. and Thomas, J. A. Elements of information theory, 1991 (John Wiley, New York, NY, USA).

25 Mallat, S. A wavelet tour of signal processing, 2nd edition, 1998 (Academic Press, Boston, MA, USA).

26 Chin, S., Ray, A., and Rajagopalan, V. Symbolic time series analysis for anomaly detection: a comparative evaluation. Signal Process., 2005, 85(9), 1859-1868.

27 Gupta, S., Ray, A., and Keller, E. Symbolic time series analysis of ultrasonic data for early detection of fatigue damage. Mech. Syst. Signal Process., 2007, 21(2), 866-884.

28 Simani, S., Fantuzzi, C., and Patton, R. Model-based fault diagnosis in dynamical systems using identification techniques, 2003 (Springer-Verlag, London, UK).
29 Diao, Y. and Passino, K. M. Stable fault-tolerant adaptive fuzzy/neural control of a turbofan engine. IEEE Trans. Control Syst. Technol., 2001, 9(3), 494-509.

30 Parker, K. I. and Guo, T. H. Development of a turbofan engine simulation in a graphical simulation environment. In JANNAF Aero-Propulsion Subcommittee Meeting, Destin, FL, USA, 2002.

31 Tolani, D. K., Yasar, M., Ray, A., and Yang, V. Anomaly Detection in aircraft gas turbine engines. J. Aerosp. Comput. Info. Commun., 2006, 3(2), 44-51.

32 Beck, C. and Schögel, F. Thermodynamics of chaotic systems: an introduction, 1993 (Cambridge University Press, Cambridge, UK).

33 Badii, R. and Politi, A. Complexity, hierarchical structures and scaling in physics, 1997 (Cambridge University Press, Cambridge, UK).

34 Daw, C. S., Finney, C. E. A., and Tracy, E. R. A review of symbolic analysis of experimental data. Rev. Sci. Instrum., 2003, 74(2), 915-930.

35 Kennel, M. B. and Buhl, M. Estimating good discrete partitions from observed data: symbolic false nearest neighbors. Phys. Rev. Lett., 2003, 91 (8), 084102.

36 Sarkar, S., Yasar, M., Gupta, S., Ray, A., and Mukherjee, K. Fault detection and isolation in aircraft gas turbine engines. Part 2. Validation on a simulation test bed. Proc. IMechE, Part G: J. Aerospace Engineering, 2008, 222(G3), 319-330 (this issue).

37 Ray, A. Signed real measure of regular languages for discrete-event supervisory control. Int. J. Control, 2005, 78(12), 949-967.

38 Gupta, S., Ray, A., and Mukhopadhyay, A. Anomaly detection in thermal pulse combustors using symbolic time series analysis. Proc. IMechE, Part I: J. Systems and Control Engineering, 2006, 220(I5), 339-351.

39 Bapat, R. B. and Raghavan, T. E. S. Nonnegative matrices and applications, 1997 (Cambridge University Press, Cambridge, UK).

40 Rajagopalan, V., Chakraborty, S. and Ray, A. Estimation of slowly-varying parameters in nonlinear systems via symbolic dynamic filtering. Signal Process., 2008, 88(2), 339-348.

\section{APPENDIX}

\section{Notation}

C

$D \quad$ window length on a symbolic sequence

$m \quad$ number of components of a system

$\mathcal{M}$ mapping from the phase space to the symbol space

$n \quad$ number of states of the finite state machine

$\boldsymbol{p} \quad$ state probability vector

$p_{i} \quad$ probability of the $i t h$ state

$P(\bullet) \quad$ probability of the event $\bullet$

$\boldsymbol{q}$ reference state probability vector 
$s \quad$ random variable taking values in $\Sigma$

$S \quad$ Shannon entropy of the symbol sequence

fast time

slow time

component input vector

state vector in the phase space

state vector at (fast) time

instant $j$

initial state vector

component output vector

nominal component model output vector

$\alpha, \beta \quad$ symbols belonging to $\Sigma$

$\delta(\bullet, \star) \quad$ state transition mapping

$\eta$ $\theta$ parameter vector varying in the slow time scale

$\lambda$ eigenvalue of the state transition matrix

$\mu \quad$ anomaly measure

$\xi_{j} \quad j$ th state of the finite state machine

$\Xi$ set of all states of the finite state machine

$\pi_{j k} \quad$ probability of the transition from states $\xi_{j}$ to $\xi_{k}$

$\Pi \quad$ state transition matrix

$\sigma \quad$ symbol on a symbolic sequence

$\Sigma \quad$ alphabet (i.e. set of symbols)

$|\Sigma| \quad$ alphabet size of the partition

$\tau$ slow time

$\Phi_{i} \quad$ partition cell of the phase space

$\Omega \quad$ compact region in the phase space 